\title{
Gender Differences in the Prevalence and Experience of Sexual Harassment of Internal Medicine Providers by Patients
}

J Gen Intern Med 36(11):3598-600 DOI: $10.1007 /$ s11606-020-06473-y

(C) Society of General Internal Medicine (This is a U.S. government work and not under copyright protection in the U.S.; foreign copyright protection may apply) 2021

$\mathrm{S}$ exual harassment of health care providers by colleagues and supervisors has received considerable attention. However, few studies provide information on provider harassment by patients and most focus on women providers. ${ }^{1-6} \mathrm{Up}$ to $76 \%$ of women providers have been sexually harassed by a patient or patient's family member. We investigated the prevalence of sexual harassment by patients among both men and women providers and the degree the various behaviors were bothersome.

\section{METHODS}

We conducted an anonymous, online survey at a Midwestern VA Medical Center between April and June of 2019 querying medicine faculty and internal medicine resident rotators. The survey was developed after literature review and expert input. We classified harassment as physical and non-physical (verbal). ${ }^{3}$ We asked whether behaviors were considered to be sexual harassment, whether participants had experienced the behavior, and how bothersome these experiences were (5-point scale from 1: not at all to 5: extremely bothered). The survey did not specify harasser gender and is available upon request. Our IRB approved this study. We assessed for differences using chi-square, $t$ tests, or the Mann-Whitney $U$ test, as appropriate (STATA v. 16.0).

\section{RESULTS}

The response rate was $78 \%$ for faculty and $41 \%$ for residents. Among the 111 respondents, most were White (54\%), women $(60 \%)$, and faculty members $(57 \%$, Table 1$)$. The male respondents were similar in race to women, though were more likely to

Received July 30, 2020

Accepted December 14, 2020

Published online February 2, 2021 be faculty, older, with more years in practice (Table 1). Men and women generally agreed on which behaviors were sexual harassment (Table 2), although women were more likely to consider being asked if they are married (49\% vs. $28 \%, p=0.04$ ) or compliments on dress/body $(88 \%$ vs. $67 \%, p=0.02)$ to be harassment. There were no differences in type or race of providers and attitudes about what constitutes sexual harassment.

Overall, ninety-nine providers (89\%) reported experiencing sexual harassment; verbal was more common than physical harassment $(89 \%$ vs. $56 \%, p<0.001)$. Women more commonly experienced harassment than men (97\% vs. $77 \%, p=$ 0.001 , Table 2 ). There was no difference by type of provider (resident vs. faculty) in the likelihood of experiencing verbal (92 vs. $87 \%, p=0.46$ ) or physical (61 vs. $71 \%, p=0.21$ ) harassment, except residents were more likely to be mistaken for a nurse $(81 \%$ vs. $41 \%, p=0.007)$. There was also no difference in harassment experience by provider race (White vs. non-White, verbal: $87 \%$ vs. $92 \%, p=0.35$, physical: $60 \%$ vs. $53 \%, p=0.45$ ).

Most verbal harassment behaviors were experienced as similarly uncomfortable by men and women, although women were more bothered by being mistaken as a nurse (2.6 vs. 1.6, $p<0.001$ ), being asked if they were married (2.3 vs. $1 ., p=$ 0.01 ), and comments on their figure or dress (3.6 vs. 2.9, $p=$ 0.007 , Table 2). For both men and women, physical sexual harassment was more bothersome than verbal harassment (5.3 vs. 3.5, $p<0.001)$. Most categories of physical harassment were more bothersome for women than men including leering, standing too close, unwanted physical contact, and stalking (Table 2). There was no difference in discomfort by provider type or race, except residents were more bothered by being

Table 1 Participant Characteristics

\begin{tabular}{llll}
\hline \hline Characteristic & $\begin{array}{l}\text { Male }(\boldsymbol{n}= \\
\mathbf{4 4})\end{array}$ & $\begin{array}{l}\text { Female }(\boldsymbol{n}= \\
\mathbf{6 7})\end{array}$ & $\boldsymbol{p}$ \\
\hline Age, median (IQR) & $47(33.5-59)$ & $34(29-51)$ & 0.003 \\
Race & $29(66 \%)$ & $31(46 \%)$ & 0.16 \\
$\quad$ White & $1(3 \%)$ & $5(7 \%)$ & \\
African-American & $7(16 \%)$ & $9(13 \%)$ & \\
Asian & $8(18 \%)$ & $22(32 \%)$ & \\
Other & $18.4(12.1)$ & $10.5(11.2)$ & $<$ \\
Years MD, mean (SD) & & & 0.001 \\
Provider type & $14(32 \%)$ & $34(51 \%)$ & 0.05 \\
$\quad$ Internal medicine & & & \\
residents & $30(68 \%)$ & $33(49 \%)$ & \\
Faculty & &
\end{tabular}


Table 2 Perception, Experience, and Discomfort with Sexual Harassment by Patients

\begin{tabular}{|c|c|c|c|c|c|c|c|c|c|}
\hline \multirow[t]{2}{*}{$\overline{\text { Behavior }}$} & \multicolumn{3}{|c|}{ Is this sexual harassment? } & \multicolumn{3}{|c|}{ Have you experienced this behavior? } & \multicolumn{3}{|c|}{ How bothersome is it $^{\#}$ ? (mean) } \\
\hline & $\begin{array}{l}\text { Female }(n= \\
\text { 57) }\end{array}$ & $\begin{array}{l}\text { Male }(n= \\
\text { 35) }\end{array}$ & $p$ & $\begin{array}{l}\text { Female }(n= \\
57)\end{array}$ & $\begin{array}{l}\text { Male }(n= \\
\text { 35) }\end{array}$ & $p$ & $\begin{array}{l}\text { Female }(n= \\
\text { 57) }\end{array}$ & $\begin{array}{l}\text { Male }(n= \\
\text { 35) }\end{array}$ & $p$ \\
\hline \multicolumn{10}{|c|}{ Verbal (nonphysical) behaviors } \\
\hline Mistaken for nurse & $11(16 \%)$ & $4(9 \%)$ & 0.27 & $56(84 \%)$ & $8(18 \%)$ & $\begin{array}{l}< \\
0.001\end{array}$ & 2.6 & 1.6 & $\begin{array}{l}<.001 \\
0.001\end{array}$ \\
\hline Asked if married & $27(40 \%)$ & $11(25 \%)$ & 0.13 & $49(73 \%)$ & $24(55 \%)$ & 0.04 & 2.3 & 1.8 & 0.01 \\
\hline Sexist jokes & $50(75 \%)$ & $36(82 \%)$ & 0.38 & $38(57 \%)$ & $17(39 \%)$ & 0.06 & 3.4 & 3.2 & 0.40 \\
\hline Derogatory statements & $47(70 \%)$ & $36(82 \%)$ & 0.17 & $44(66 \%)$ & $15(34 \%)$ & 0.001 & 3.6 & 3.5 & 0.71 \\
\hline $\begin{array}{l}\text { Comments on } \\
\text { body/dress }\end{array}$ & $62(93 \%)$ & $24(55 \%)$ & 0.001 & $53(79 \%)$ & $12(28 \%)$ & $\begin{array}{l}< \\
0.001\end{array}$ & 3.6 & 2.9 & 0.007 \\
\hline Flirtation & $40(60 \%)$ & $23(52 \%)$ & 0.44 & $49(73 \%)$ & $8(18 \%)$ & $\begin{array}{l}< \\
0.001\end{array}$ & 3.2 & 3.1 & 0.62 \\
\hline Offensive language & $58(87 \%)$ & $34(77 \%)$ & 0.20 & $19(28 \%)$ & $1(2 \%)$ & $\begin{array}{l}< \\
0.001\end{array}$ & 4.0 & 3.9 & 0.85 \\
\hline Sexual interest & $62(93 \%)$ & $37(84 \%)$ & 0.16 & $35(52 \%)$ & $6(13 \%)$ & $\begin{array}{l}< \\
0.001\end{array}$ & 4.3 & 4.0 & 0.15 \\
\hline Offensive pictures & $58(87 \%)$ & $37(84 \%)$ & 0.72 & $10(15 \%)$ & $6(13 \%)$ & 0.85 & 4.3 & 4.1 & 0.44 \\
\hline $\begin{array}{l}\text { Unwanted } \\
\text { compliment }\end{array}$ & $43(64 \%)$ & $21(47 \%)$ & 0.09 & $51(76 \%)$ & $2(5 \%)$ & $\begin{array}{l}< \\
0.001\end{array}$ & 3.0 & 2.6 & 0.14 \\
\hline Request for date & $52(78 \%)$ & $28(64 \%)$ & 0.11 & $18(27 \%)$ & $6(13 \%)$ & 0.10 & 3.9 & 3.7 & 0.18 \\
\hline $\begin{array}{l}\text { Sexual proposition } \\
\text { (implicit) }\end{array}$ & $62(93 \%)$ & $41(93 \%)$ & 0.90 & $15(22 \%)$ & $1(2 \%)$ & 0.003 & 4.5 & 4.3 & 0.14 \\
\hline \multicolumn{10}{|l|}{ Physical behaviors } \\
\hline Leering & $55(82 \%)$ & $32(73 \%)$ & 0.24 & $46(69 \%)$ & $2(5 \%)$ & $\begin{array}{l}< \\
0.001\end{array}$ & 3.6 & 3.0 & 0.007 \\
\hline Standing too close & $48(72 \%)$ & $31(70 \%)$ & 0.89 & $46(69 \%)$ & $8(18 \%)$ & $\begin{array}{l}< \\
0.001\end{array}$ & 3.6 & 3.1 & 0.04 \\
\hline $\begin{array}{l}\text { Unwanted physical } \\
\text { contact }\end{array}$ & $61(91 \%)$ & $44(100 \%)$ & 0.06 & $23(34 \%)$ & $2(5 \%)$ & $\begin{array}{l}< \\
0.001\end{array}$ & 4.7 & 4.5 & 0.11 \\
\hline Exposure of body part & $60(90 \%)$ & $43(98 \%)$ & 0.10 & $23(34 \%)$ & $3(7 \%)$ & 0.001 & 4.5 & 4.4 & 0.31 \\
\hline $\begin{array}{l}\text { Sexual proposition } \\
\text { (explicit) }\end{array}$ & $60(90 \%)$ & $44(100 \%)$ & 0.03 & $89(13 \%)$ & $2(5 \%)$ & 0.13 & 4.8 & 4.5 & 0.01 \\
\hline Sexual bribery & $60(90 \%)$ & $43(98 \%)$ & 0.10 & $2(3 \%)$ & $0(0 \%)$ & 0.52 & 5.0 & 4.8 & 0.006 \\
\hline Stalking & $57(85 \%)$ & $43(98 \%)$ & 0.03 & $6(7 \%)$ & $0(0 \%)$ & 0.08 & 5.0 & 4.7 & 0.008 \\
\hline
\end{tabular}

Not all questions were answered

\#Bothersome rated as 1: not at all; 2: bothered a little; 3: moderately bothered; 4: bothered quite a bit; 5: extremely bothered

mistaken for a nurse $(2.7$ vs. $1.5, p<0.001)$. Among those who reported experiencing harassment, being mistaken for a nurse (2.6 vs. $1.6, p<0.001)$, unwanted compliments (3.1 vs. $2.6, p=0.04)$, physical contact $(4.8$ vs. $4.3, p=0.04)$, and explicit sexual propositions ( 4.8 vs. $4.2, p=0.003$ ) were rated as more bothersome than among those who had not experienced it. The remaining behaviors were rated similarly, regardless of respondent experience.

\section{DISCUSSION}

Consistent with previous literature, we found that sexual harassment of providers by patients is common. Women were more likely to experience all forms of harassment. While men reported high levels of verbal harassment, they infrequently experienced physical harassment. For both genders, physical harassment was considered more bothersome than verbal harassment, and women reported more behaviors as bothersome. Our study has several limitations including small sample size, single center, reliance of survey data, and no framing of the time period for survey questions. Our data adds to the growing body of literature demonstrating the ongoing problem of sexual harassment by patients. Given the agreement between men and women on what constitutes harassment, institutions can help better define the problem to allow for the development of curricular and health care institutional interventions to address this problem.

Jeffrey L. Jackson, MD MPH ${ }^{1,2}$

Amy Farkas, $M D M S c^{1,2}$

Kathlyn Fletcher, MD MSc ${ }^{1,2}$

Cynthia Kay, MD MSc ${ }^{1,2}$

Julie L. Machen, $M D^{3}$

Sarah Nickoloff, $M D^{1,2}$

Cecilia Scholcoff, $M D M P H^{1,2}$

${ }^{1}$ Clement J. Zablocki VA Medical Center,

Milwaukee, USA

${ }^{2}$ Medical College of Wisconsin,

Milwaukee, USA

${ }^{3}$ Dell Medical School at the University of Texas,

Austin, USA

Corresponding Author: Jeffrey L. Jackson, MD MPH; Clement J. Zablocki VA Medical Center, Milwaukee, USA (e-mail:jjackson@mcw.edu). 


\section{Compliance with Ethical Standards:}

Conflict of interest: The authors declare that they have no conflict of interest.

\section{REFERENCES}

1. Blumenreich P. Sexual harassment of female physicians by male patients. J Ky Med Assoc 1993;91(7):291-292.

2. Connolly MJ. Results of the Committee on Women Physicians' survey. Minn Med 1993;76(9):21-23.

3. Phillips $\mathbf{S}$. Sexual harassment of female physicians by patients. What is to be done? Can Fam Physician 1996;42:73-78.
4. Phillips SP, Schneider MS. Sexual harassment of female doctors by patients. N Engl J Med 1993;329(26):1936-1939.

5. Jenner S, Djermester P, Prügl J, Kurmeyer C, Oertelt-Prigione $\mathbf{S}$. Prevalence of Sexual Harassment in Academic Medicine. JAMA Intern Med 2019;179(1):108-111.

6. Vargas EA, Brassel ST, Cortina LM, Settles IH, Johnson TRB, Jagsi R. \#MedToo: A Large-Scale Examination of the Incidence and Impact of Sexual Harassment of Physicians and Other Faculty at an Academic Medical Center. $J$ Women's Health (Larchmt). 2020;29(1):13-20.

Publisher's Note: Springer Nature remains neutral with regard to jurisdictional claims in published maps and institutional affiliations. 